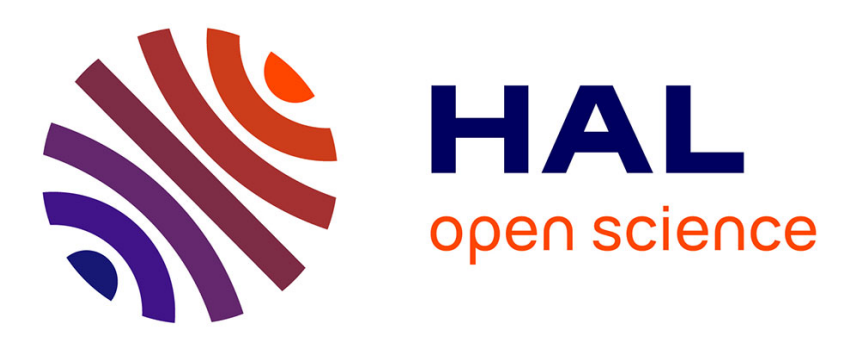

\title{
Un " pèlerinage à l'oracle " : Edith Wharton, Henry James et la patrimonialisation de la maison de George Sand à Nohant
}

Elizabeth Emery

\section{- To cite this version:}

Elizabeth Emery. Un " pèlerinage à l'oracle " : Edith Wharton, Henry James et la patrimonialisation de la maison de George Sand à Nohant. Culture et Musées, 2019, 34, pp.107-138. 10.4000/culturemusees.3819 . hal-03254164

\section{HAL Id: hal-03254164 \\ https://hal.science/hal-03254164}

Submitted on 8 Jun 2021

HAL is a multi-disciplinary open access archive for the deposit and dissemination of scientific research documents, whether they are published or not. The documents may come from teaching and research institutions in France or abroad, or from public or private research centers.
L'archive ouverte pluridisciplinaire HAL, est destinée au dépôt et à la diffusion de documents scientifiques de niveau recherche, publiés ou non, émanant des établissements d'enseignement et de recherche français ou étrangers, des laboratoires publics ou privés. 


\section{OpenEdition}

Journals

\section{Culture \& Musées}

Muséologie et recherches sur la culture

$34 \mid 2019$

Maisons-musées. La patrimonialisation des demeures des illustres

Dossier

\section{Un « pèlerinage à l'oracle »: Edith Wharton, Henry James et la patrimonialisation de la maison de George Sand à Nohant}

A "Pilgrimage to the Oracle": Edith Wharton, Henry James and the Patrimonialisation of La Maison de George Sand in Nohant

Una «peregrinación al oráculo». Edith Wharton, Henry James y la patrimonialización de la casa de George Sand en Nohant

\section{ELIZABETH EMERY}

p. $107-138$

https://doi.org/10.4000/culturemusees.3819

\section{Résumés}

Français English Español

En prenant pour point de départ les articles et livres que les écrivains américains Edith Wharton et Henry James ont consacrés à la maison de George Sand à Nohant, cet article se propose d'examiner comment, et avec quelle complexité, les publications de célébrités peuvent renforcer pour le public l'importance culturelle des maisons d'écrivains, une catégorie de maison-musée dotée d'une valeur mémorielle particulière. La visite de la maison de Sand par Wharton soulève aussi des questions propres à la mise en scène d'autres maisons-musées consacrées aux femmes.

Using popular writings about George Sand's "château" in Nohant published by American writers Edith Wharton and Henry James as case study, this article argues for the important role played by celebrity publications in increasing the public's awareness of the cultural importance of writerhouses, a particularly complex memorial subset of the house-museum. Wharton's visit to Sand's home also draws attention to the dangers inherent in exhibits highlighting the domestic life of women.

Tomando como punto de partida los artículos y libros que los escritores estadounidenses Edith Wharton y Henry James han dedicado a la casa de George Sand en Nohant, este artículo pretende examinar cómo y con qué complejidad las publicaciones de celebridades pueden fortalecer frente al público, la importancia cultural de las casas de los escritores, una categoría de casa museo con 
un valor conmemorativo particular. La visita de Wharton a la casa de Sand, también plantea interrogantes en torno a la puesta en escena de otras casas-museo dedicadas a mujeres.

\section{Entrées d'index}

Mots-clés : littérature, imagination, lieu de mémoire, culture de masse, femmes de lettres, Wharton (Edith), James (Henry), Sand (George)

Keywords: literature, imagination, sites of memory, popular culture, women writers, Wharton (Edith), James (Henry), Sand (George)

Palabras clave: literatura, imaginación, lugar de la memoria, cultura de masas, mujeres de letras, Wharton (Edith), James (Henry), Sand (George)

\section{Notes de la rédaction}

Manuscrit reçu le 20 mars 2019

Version révisée reçue le 25 octobre 2019

Article accepté pour publication le 9 novembre 2019

\section{Texte intégral}

En 1904, l'écrivaine américaine Edith Wharton (1862-1937) traversa la France au volant de sa première voiture pendant ses vacances familiales : de Nice à Pau, de Limoges à Amiens, de Paris à Poitiers ${ }^{1}$. En route, elle prit des notes sur les sites culturels rendus accessibles par ce nouveau mode de transport et partagea ensuite ses impressions sur ces visites d'un nouveau type dans une série d'articles intitulée $A$ Motor-Flight through France ${ }^{2}$ (littéralement « un vol par moteur », traduit en français par La France en automobile [Wharton, 2015]). Ce récit, considéré par une génération de touristes anglo-américains comme un guide culturel du patrimoine français, doit son influence à la façon dont il souligne les particularités de chaque région : le voyage en automobile dévoile l'importance de la géographie française pour le développement de l'architecture, de la cuisine et même l'existence d'un caractère propre aux provinces 3 . Ce qui surprend dans ce récit consacré largement aux églises, châteaux et forteresses, c'est l'emphase qu'elle met sur le passage consacré à une résidence privée non encore repérée par d'autres guides - la maison de George Sand à Nohant - et à laquelle l'écrivaine consacre deux chapitres ${ }^{4}$. Au contraire d'autres guides anglophones comme celui d'Henry James (A Little Tour in France) dont nous discuterons plus bas, Wharton mentionne très peu d'écrivains dans A Motor-Flight5.

2 Encore plus surprenant, Wharton présente le « château de George Sand »- que les riverains ne connaissent même pas lorsqu'elle essaie d'y accéder en 1906 - comme un " pèlerinage à l'oracle » ${ }^{6}$. Élevée sur le même plan dans son texte que les monuments historiques officiellement reconnus (Notre-Dame de Reims et Carcassonne, par exemple), la simple maison de George Sand gagne ainsi une nouvelle visibilité internationale. En révélant l'existence d'un lieu méconnu du public, elle en facilite l'accès et en expose à la fois l'intérêt et les modalités de la visite. Il s'agit, en fait, de la première étape - « communiquer le patrimoine » - identifiée par Jean Davallon comme nécessaire à la patrimonialisation? .

Dans cet article, nous prenons comme point de départ les textes - articles, essais, lettres, mémoires - publiés par Wharton après son voyage à Nohant afin d'examiner la manière dont des écrits littéraires peuvent générer une activité touristique qui alimente la «mentalité » identifiée par Françoise Choay comme nécessaire au développement des concepts de patrimoine et de monument historique ${ }^{8}$. Dans une première partie, nous examinerons la renommée internationale de Sand, qui motiva la visite de Wharton et sa revendication de la maison d'écrivain comme un lieu de pèlerinage à la hauteur d'autres destinations littéraires comme les tombes de figures essentiellement masculines : Pétrarque, Dante, Rousseau ou Keats ${ }^{9}$. Une deuxième partie considèrera la façon dont Wharton a anticipé et fait valoir, dès 1906, l'intérêt littéraire, touristique et ethnographique de cette maison si excentrée (dans le Berry), célèbre aujourd'hui non seulement pour ses illustres visiteurs (Sand, Chopin, Balzac, Delacroix, Liszt, Flaubert 
et Dumas fils), mais aussi pour sa riche collection de manuscrits, marionnettes et objets authentiques qui témoignent de la vie à la campagne au XIXe siècle. À la lumière des procédés identifiés par Davallon comme nécessaires à la patrimonialisation (Cf. note 7), une troisième partie évaluera les choix architecturaux et muséographiques décidés aux XXe et XXIe siècles pour faire entrer la maison de Sand - celle d'une femme - dans une rhétorique patrimoniale traditionnellement réservée aux « grands hommes ».

\section{La communication du patrimoine. George Sand, célébrité internationale}

On connaît la renommée de George Sand en France au courant du XIXe siècle, mais on connaît bien moins - à l'époque comme aujourd'hui - sa popularité à l'étranger ${ }^{10}$ : "L'influence de George Sand sur la société européenne, sans en excepter la société russe, fut immense de 1835 à 1855 ", écrit sa première biographe critique. "On disait : "le siècle de George Sand" comme on disait : "le siècle de Byron" " (Karénine, 1899 : I, 11). Sand fut particulièrement populaire parmi les Américains lettrés comme Edith Wharton et son ami Henry James, qui a publié huit essais consacrés aux écrits de Sand de 1868 à $1914^{11}$. Elle fut une pierre de touche de leur amitié. Ensemble, ils la lurent en français (surtout des textes de nature autobiographique comme Histoire de ma vie $(H D M V)$, Elle et Lui et des collections de lettres publiées après la mort de Sand [1876] puis celle de sa fille, Solange [1899]), discutèrent et s'envoyèrent (avec des passages soulignés au crayon) les nouvelles publications sur Sand. Ils partagèrent le rêve de visiter sa lointaine villégiature de Nohant ${ }^{12}$.

5 Cette appréciation mutuelle semble dater d'une visite de James chez Wharton en juin $1905 \mathrm{au}$ "Mount » (désormais musée américain connu sous le nom de "Edith Wharton's Home » [la maison d'Edith Wharton]), à Lenox, dans l'ouest du Massachusetts ${ }^{13}$. Passant leurs soirées à lire à haute voix le premier volume de la biographie de Karénine, ils finirent par doter - de façon ludique - les différentes voitures achetées par le mari de Wharton cet été-là de surnoms sandiens : ils découvrirent le paysage des montagnes Berkshire dans la magnifique mais peu fiable « Alfred », la petite mais infatigable « George » et la puissante « Pagello » (du nom du médecin de Musset qui remplaça ce dernier dans les affections de Sand) ${ }^{14}$. Leur rêve de visiter la maison de Sand ne résulte donc pas d'un simple culte de la personnalité mais d'une profonde connaissance de ses œuvres qu'ils souhaitent mieux comprendre en visitant les lieux que l'écrivaine elle-même avait identifiés comme signifiants (" Il est difficile de parler de Nohant sans dire quelque chose qui ait rapport à ma vie présente ou passée $\left.e^{15} »\right)$.

$6 \quad$ Lorsque Wharton annonce, en juillet 1906, qu'elle a pu visiter Nohant en automobile, la jalousie de James - qui rêve d'y aller depuis des années ${ }^{16}$ - déborde de ses lettres (2 juillet 1906) :

\footnotetext{
« Depuis des semaines je suis réellement dans l'état estropié - la plaie saignante au côté - produit par le coup Parthian de votre dernière lettre - le javelot que vous avez lancé en partant, de cette vision indicible de Nohant dont la vision illuminée m'échappe si misérablement toutes ces longues années (sans moteur) [...] la brume dorée de votre Nohant [...] la toute première question dont je vous accablerai sera de votre aventure et impression de Nohant - dont je brûle et désire ardemment des détails. [...] À penser que vous avez vu La Châtre ! [...] Mais il [le javelot] restera là à m'empoisonner jusqu'à ce que vous écriviez - que vous IMPRIMIEz, “fassiez” le lieu, que m'envoyiez toute l'impression sous pression de publication imminente. Car vous le faites, vous l'avez fait. Vous ne pouvez pas ne pas le faire. Je meurs d'envie et je languis. Écrivez-moi que cet acte de piété est même déjà fait [...]. Il n’y a, vous le savez, aucun récit (de l'impression du lieu) d'une autorité ou valeur quelconque à part celle de George elle-même. Comment vous avez dû tous les sentir [smelt]17! »
} Parthian », javelot, « brume dorée ») que pour son insistance sur le fait que Wharton 
fixe Nohant par écrit et surtout qu'elle publie ses impressions. Comme James le dira dans une lettre de 1914, Nohant fut autrefois "si timide et isolé » [so shy and remote], que même les plus motivés (comme lui) n'arrivaient pas à y accéder ${ }^{18}$. Sand elle-même évoque le long et pénible voyage de Paris à Nohant en calèche (de trois à quatre jours au début du siècle) dans de nombreux passages de $H D M V$ où elle insiste sur les dangers (brigands, boue, animaux sauvages). Selon les estimations de Perrot, le voyage ne prendra que trente heures (minimum) en 1840, et 24 en 1855 grâce au chemin de fer ${ }^{19}$. Le fait d'entreprendre ce voyage compliqué et surtout de documenter le lieu et d'en expliquer les modalités de sa visite, consiste donc pour James en un "acte de piété " qui rendra hommage à ce " lieu saint ${ }^{20}$ en le faisant mieux connaître. Il adopte donc le langage quasi religieux de pèlerinage culturel de plus en plus courant au XIXe siècle (Hendrix, 2008), ce qui rehausse encore son propre prestige ainsi que celui de Wharton : ces Américains se comptent parmi les élus qui connaissent Nohant.

8 Le « martyre » que subit James en lisant le récit de Wharton se comprend par le fait qu'il a été un des pionniers du récit touristique littéraire : A Little Tour in France fut écrit en 1884 pour faire connaître la province française aux lecteurs anglophones, consommateurs avides de guides sur la France (on dénombre quelque 600 guides publiés au XIXe siècle) ${ }^{21}$. Ce texte extrêmement populaire partage les impressions enregistrées par James pendant les six semaines qu'il avait passées à découvrir le patrimoine français en train, en omnibus et en calèche : de Tours, La Rochelle et Toulouse, il a longé la Méditerranée (Arles, Avignon) avant de remonter jusqu'à Dijon. Ses notes lient la province française à ses traditions culturelles et littéraires, évoquant Tours par les écrits de Balzac et Stendhal, par exemple, Chenonceau par ceux de Rousseau, Narbonne et Tarascon par ceux de Daudet, Fontaine-de-Vaucluse par la présence de Pétrarque, Mâcon par celle de Lamartine. Ce livre, qui eut un succès notable et fut longtemps considéré comme le meilleur guide sur la France ${ }^{22}$, établit pour les touristes anglophones - 100 ooo par an dans les années 1890 (Schriber, 1991 : xxxix) - une espèce de carte des lieux littéraires français, un siècle avant celle fixée en 1997 par la Fédération des maisons et des patrimoines littéraires ${ }^{23}$.

$9 \quad$ Malgré sa familiarité avec les histoires de la vie à Nohant, James, qui n'était pas riche comme Wharton, n’a jamais pu accéder à cette maison si excentrée. Lorsque les Wharton projettent d'embarquer pour de nouveaux voyages en automobile au printemps de 1907 et proposent de l'amener avec eux, il accepte à condition de passer par Nohant ${ }^{24}$. Du 20 mars au 12 avril 1907, ils font près de 3600 kilomètres en automobile, de Paris en passant par Chartres, Blois, Nohant, Poitiers, Chauvigny, Angoulême, Bordeaux, Pau, Toulouse Carcassonne, Hyères, Avignon, Sens et Fontainebleau ${ }^{25}$. Wharton semble avoir tout fait pour rendre le voyage de son ami mémorable, y compris en organisant une visite de l'intérieur de la maison de George Sand. Ce double passage à Nohant par Wharton - en famille et ensuite avec James explique donc la proéminence de ce lieu littéraire parmi les monuments historiques figurant dans A Motor-Flight : un premier chapitre, décrit de l'extérieur, fut sérialisé dans The Atlantic Monthly (numéro de janvier 1907), et le second, après sa visite avec James, évoque l'intérieur (numéro de février 1908).

10 Dans ces articles, elle « fait le lieu », comme l'avait demandé James. Mais « faire le lieu » ne consiste pas à donner de simples notations pratiques qui caractérisent les guides touristiques comme ceux de Murray, Joanne ou Baedeker, que James et Wharton ont employés pour se situer lors de leurs déplacements, mais à produire des ekphrasis, c'est-à-dire des portraits littéraires du lieu. Ils servent donc beaucoup plus à éveiller l'intérêt des lecteurs par leurs descriptions stylisées qu'à renseigner les visiteurs sur les modalités du voyage. D'où le curieux commentaire de James concernant les odeurs lorsqu'il parle des compagnons de Sand dans sa lettre à Wharton : « vous avez dû tous les sentir».

11 Le rapport personnel de ces écrivains inspire un double mouvement de pèlerinage littéraire : les lecteurs de James et Wharton vont découvrir et valider le patrimoine historique et littéraire français en suivant les pas de leurs écrivains bien-aimés et en partageant ensuite leurs propres impressions ${ }^{26}$. Mais James et Wharton suivent euxmêmes les illustres qui les ont devancés, faisant de leurs récits de véritables palimpsestes de lecture et d’interprétation. Tiphaine Samoyault note que « la mémoire 
de la littérature se joue sur trois niveaux qui ne se recouvrent jamais entièrement : la mémoire portée par le texte, la mémoire de l'auteur et la mémoire du lecteur » (2004: 111). On pourrait ajouter " la mémoire du lieu », où les trois autres éléments entrent en contact. James, par exemple, avance l'idée qu'un visiteur peut « communier » avec de grands écrivains du passé en fréquentant des lieux peu changés depuis leur passage terrestre (1884:226); ce contact spatial aiderait à mieux comprendre ses devanciers et leur œuvre, et à mieux s'inscrire dans leur lignage littéraire ${ }^{27}$.

12 Nohant est un lieu idéal pour ce genre de communion mémorielle car il fut, même du vivant de Sand, « un lieu de rencontre, un adoubement, un sacrement [...]. Y aller est un pèlerinage, une initiation, l'intronisation dans la société choisie que George Sand rêve de cristalliser, de cimenter autour de sa maison » (Perrot, 2018 : 98). En pèlerins modernes, Wharton et James suivent les traces de Sand, celle qui, la première, a " communiqué » cet intérêt culturel de Nohant : " Est-il beaucoup de mortels qui aient eu dans leur vie le bonheur de réunir sous le même toit, presque tous les jours, pendant un mois, douze ou quinze créatures nobles et vraies, et toutes unies entre elles d'une sainte amitié ? $»^{28}$

\section{Nohant, « ville capitale de la littérature »}

Pour valider l'importance culturelle de la maison de Sand dans A Motor-Flight, Wharton célèbre Nohant à travers la renommée de ses visiteurs, insistant sur le fait que ces illustres personnages ne seraient pas venus si l'expérience n'en valait pas la peine : "Lorsqu'on considère la fatigue d'un long voyage en chemin de fer, et la crainte française des déplacements, le flot d'illustres que Paris déversait sur Nohant donne la mesure de ce que Nohant avait à offrir ${ }^{29}$. » Ainsi, Wharton imagine, lors de son passage à l'Hôtel Sainte-Catherine de Châteauroux ${ }^{30}$, les différents artistes - Liszt, SainteBeuve, Gautier, Flaubert - qui ont fréquenté la salle à manger, peu changée depuis cinquante ans, prenant leur café avant de monter dans la diligence pour rejoindre Nohant lors de leur « pèlerinage à l'oracle » (Wharton, $1908: 40$ ).

14 En exagérant la difficulté d'accès, Wharton établit par métonymie l'importance de Sand, « l'oracle », porteuse de la divine parole, l'ultime objet d'un pénible déplacement. Ce n'est donc pas Nohant, mais Sand elle-même - écrivaine, correspondante, conseillère et organisatrice de collaborations interdisciplinaires - qui constitue la destination finale. D'un coup, dans A Motor-Flight, le Berry tout entier est transformé en "Sand country », un choix qui préfigure le label employé aujourd'hui dans le Berry pour orienter les visiteurs et pour encourager le tourisme local : " le pays de George Sand ", accompagné d'une devise figurant un portrait de l'écrivain. Les chauffeurs et randonneurs peuvent suivre la « route historique George Sand » ou des « circuits George Sand » à partir de panneaux indiquant les lieux correspondant à ses romans (figure 1$)^{31}$.

Figure 1. Le pays de George Sand. 


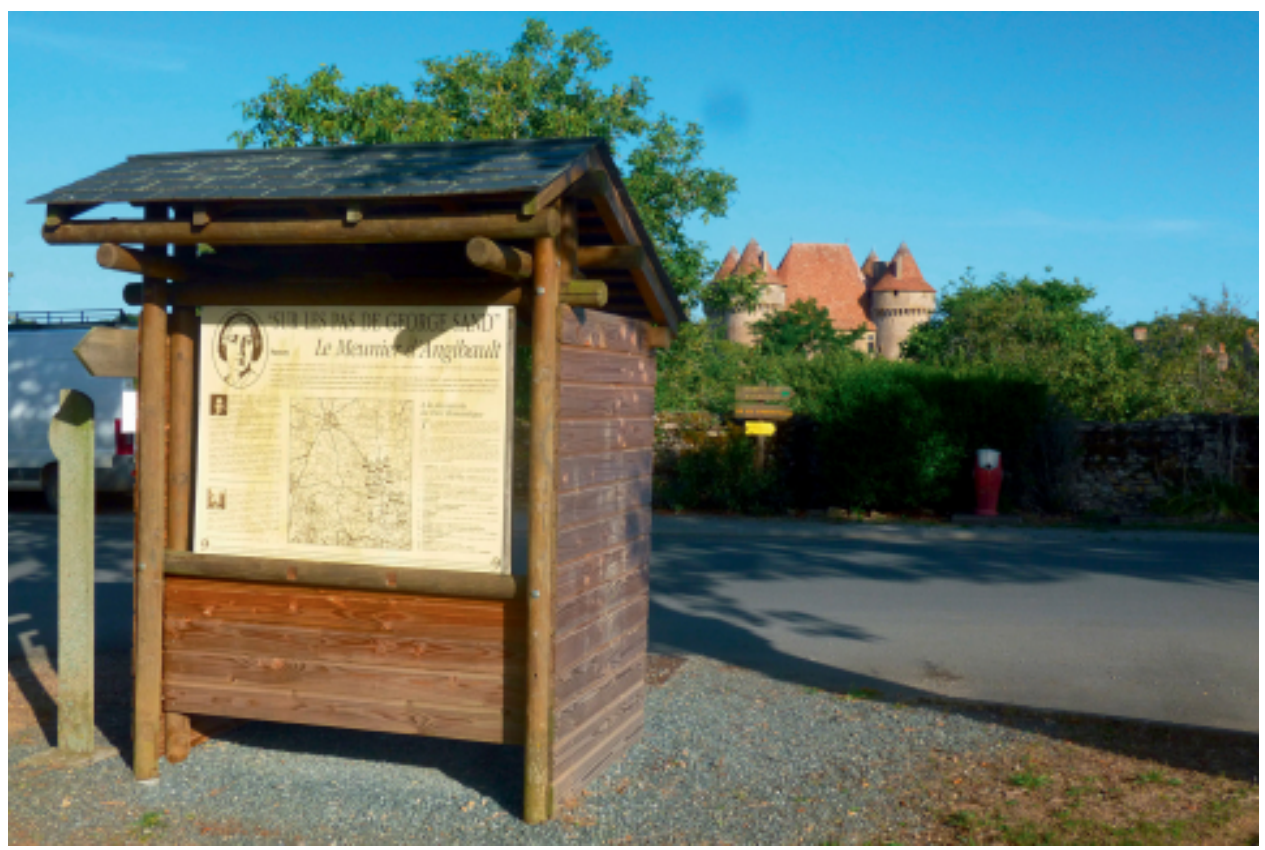

(c) Domaine public.

Wharton continue à mettre la visite à Nohant sous l'égide des célèbres visiteurs en établissant un parallèle entre sa propre description du paysage berrichon - vu de l'automobile - et ce qu'auraient vu ces grands artistes en arrivant par diligence : une campagne solitaire avant d'arriver à une simple maison beige, " une gentilhommière typique ». Aucun panneau, aucun indice pour témoigner de sa valeur patrimoniale en 1906 : la maison ne semble pas avoir changé depuis 1830. Lorsque Wharton croise une très pittoresque gardeuse d'oies, sortie tout droit des pages d'un roman comme $L a$ Mare au diable, elle l'interprète comme un point de contact entre le passé et le présent :

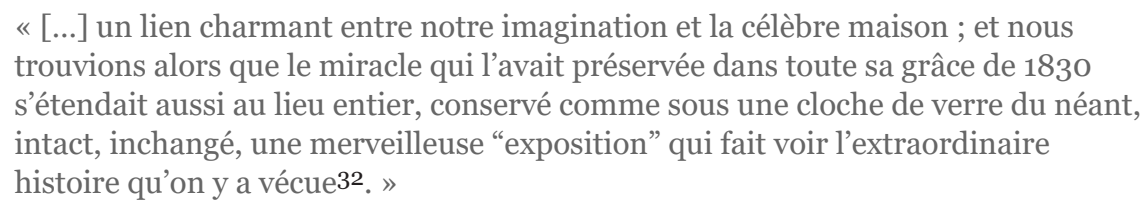

Cette référence à une " cloche de verre » rappelle à la fois la prédilection américaine pour la conservation des reliques et surtout des spécimens naturels, et la façon dont la mise en écrit par des écrivains comme Wharton sert elle-même à mettre en vitrine leurs sujets en se les appropriant, les fixant selon leur propre point de vue. Comme le dit Susan Stewart : "Le monde délicat et hermétique du souvenir est un monde de la nature idéalisé ; la nature est enlevée du domaine sauvage pour être restituée dans la sphère domestique de l'individu et de l'intérieur ${ }^{33}$. " Nohant devient, dans le texte de Wharton, à la fois un microcosme conservant un mode de vie inchangé depuis 75 ans (celui idéalisé et fixé par sa description admiratrice) et une invitation à découvrir une espèce d'exposition interactive qui fera revivre un passé maintenant disparu auquel le voyageur intrépide - et désormais motorisé - pourra accéder 34 .

À l'inverse d'une attraction d'histoire vivante, comme le «Vieux Paris » à l'Exposition universelle de 1900, Nohant ne communique qu'en filigrane l'histoire française ; « l'extraordinaire histoire » dont elle parle est celle enregistrée dans HDMV que Sand publia de 1854 à 1855 . Wharton puise donc dans « sa mémoire de texte » pour « faire le lieu », anticipant ainsi le mécanisme de ce que Pierre Nora (1997) appellera un lieu de mémoire, en l'occurrence l'importance symbolique que prend la maison dans la mémoire collective. Sans la reconnaissance de cette valeur collective liant le présent au passé, elle ne serait qu'une simple maison au bord de la route.

Cette question de valeur symbolique est au cœur de la patrimonialisation des maisons d'écrivains, comme le montre Anne Trubek dans son " guide sceptique " ( $A$ Skeptic's Guide to Writers' Houses, 2010) : pourquoi visiter ou conserver ces maisons inhabitées et souvent dénuées d'intérêt architectural ou historique en soi - un problème prononcé pour les maisons plus modernes des États-Unis ? Et à quoi bon interpréter les 
créations imaginaires d'un écrivain à la lumière d'une résidence toute matérielle35 ? Wharton reconnaît cette tension entre imagination et réalité domestique devant la maison de Sand : "Un aperçu de Nohant aide-t-il à élucider le mystère ? ou l'approfondit-il ? [...] Les deux. Car si cela aide à comprendre un aspect de la vie de George Sand, cela semble en obscurcir bien d'autres ${ }^{36}$. »Comme pour la ville de Châteauroux qui devient dans A Motor-Flight une "ville capitale de la littérature ", c'est la contradiction entre la simplicité et la « dignité » de la maison à Nohant et les scandaleux récits de $H D M V$ qui la rend si intéressante. Wharton rappelle la cohabitation mal assortie de parents illégitimes, de représentants de différentes classes sociales et professionnelles, d'amis de croyances diverses, et la présence néfaste des compagnons ivres qui font imaginer sinon une maison gothique comme celle des Hauts de Hurlevent, au moins une maison "à l'air déclassé » (1908 : 45-47). Par son style, Wharton transforme cette simple maison de campagne en témoin de l'histoire française, de toutes les activités artistiques et politiques initiées par Sand et ses visiteurs célèbres.

Les essais de Wharton et leur éloge du « château de Sand » de 1906 à 1908 ont établi sa valeur littéraire et historique pour des touristes anglo-américains ${ }^{37}$, et ont permis de partager son enthousiasme pour Nohant en France lorsqu'elle fréquentait les cercles littéraires parisiens de l'académicien Paul Bourget, son grand ami. Le moment est à propos, car Charles Spoelberch de Lovenjoul vient de léguer (1907) des reliques sandiennes (tresses de cheveux, manuscrits) à l'Institut de France ${ }^{38}$. Fortement influencé par la donation par le duc d'Aumale du domaine de Chantilly en 1897, un acte qui avait inauguré en France le don de maisons-musées, Lovenjoul spécifie que sa bibliothèque doit rejoindre celle du Musée Condé39. Léguer à l'Académie française la maison de Nohant ainsi que 100 ooo francs pour son entretien aurait donc paru logique à Gabrielle Dudevant-Sand qui, lorsqu'elle apprend en automne 1908 qu'elle a un cancer mortel (elle n'a que 41 ans), cherche à conserver les éléments matériels qu'elle n'avait pas encore communiqués à Lovenjoul ${ }^{40}$. En adoptant dans son testament le vocabulaire du pèlerinage et du culte employé par James et Wharton - « pour servir de lieu d'excursion et de pèlerinage »-, elle se montre également sensible à la valeur mémorielle de cette maison. Sa sœur, Aurore Lauth-Sand, renforcera cette volonté commémorative en insistant sur le fait que Gabrielle avait voulu « qu'ils fussent les objets d'un véritable culte : celui de l'illustre écrivain, à qui ils ont appartenu, dans un musée qui serait ainsi un véritable temple ${ }^{41} »$.

En France, la reconnaissance institutionnelle de la valeur mémorielle des maisons d'écrivains fut beaucoup plus lente qu'ailleurs en Europe : la conservation de la maison de Shakespeare date de 1847, par exemple. La Maison de Victor Hugo, ouverte par la Ville de Paris en partenariat avec les amis d'Hugo, place des Vosges, en 1903, légitime le modèle de la maison d'écrivain après quinze années de travail et divers " pèlerinages » et «musées » hugoliens établis par des individus (Emery, 2015 : 241-311). D’autres maisons d'écrivains suivent. Alexandrine Zola lègue la maison bâtie et décorée par son époux à l'Assistance publique en 1905 car elle n'avait pas les moyens de la garder, en stipulant que la maison de Médan (Yvelines) doit rester ouverte à " tous les personnages qui se présenteront pour visiter la maison de campagne en souvenir d'Émile Zola, et pour honorer la mémoire de l'illustre écrivain » (ibid. : 286-289). « Le pèlerinage à Médan » a lieu tous les ans le premier dimanche d'octobre. Comme pour la maison de Nohant, c'est la littérature - les romans de Zola écrits à Médan ainsi que ceux de ses visiteurs (Les Soirées de Médan), et les nombreuses lettres et mémoires enregistrant les activités sociales qui ont eu lieu dans cette maison de campagne (dont s'est copieusement servi Danièle Thompson en mettant en scène Cézanne et moi en 2016) - qui a renforcé une valeur patrimoniale réactualisée chaque automne par les discours politiques et littéraires télédiffusés lors du « pèlerinage à Médan »42.

«L'opérativité symbolique » (Davallon, 2006 : 135), la façon dont on communique l'importance du patrimoine au public, passe donc, pour Wharton, à travers deux éléments principaux : celui d'un centre artistique d'importance historique et celui d'un lieu littéraire, nécessaire à la compréhension des écrits de sa propriétaire. En reconnaissant la difficulté de faire valoir la littérarité d'un lieu tout matériel, elle adopte une stratégie qui persiste aujourd'hui, celle de présenter Nohant comme une ancre de la 
vie et de l'œuvre sandiennes, une maison qui lui aurait fourni la structure et le rythme nécessaires pour écrire43. Wharton, qui venait de faire construire et décorer sa propre maison de campagne ( « The Mount »), avec l'aide d'amis architectes et jardiniers (19011902), semble particulièrement bien placée pour comprendre le pouvoir d'une telle résidence sur le rythme de la vie littéraire, surtout celle des écrivaines qui ne peuvent souvent écrire qu'une fois les tâches domestiques accomplies (Sand, pour sa part, écrit de minuit à quatre heures du matin : une fois les enfants et les invités couchés) ${ }^{44}$.

\section{Maison d'écrivain, maison de femme}

En même temps, une opérativité symbolique qui privilégie la vie domestique risque de renforcer les stéréotypes de genre, surtout pour les Françaises qui, avant 1944, devaient mettre en avant leur féminité en jouant la bonne épouse, mère, ou maîtresse de maison. Effectivement, si la première visite de Wharton à Nohant en $1906 \mathrm{a}$ eu lieu sous le signe du pèlerinage littéraire, rattachant ainsi la demeure de la grande écrivaine au patrimoine historique et artistique, la seconde, en 1907, se fera sous le signe de ce que Wharton appelle les " piétés domestiques ", les décevantes réalités historiques de la femme au XIXe siècle 45 . Wharton aura du mal à réconcilier la Sand qu'elle admire l'écrivaine qui dénonce les injustices sociales - avec la bourgeoise conventionnelle que représente la figure de la « bonne dame de Nohant ». L'ambivalence de sa représentation de Sand au foyer soulève des questions propres à la mise en scène d'autres maisons-musées consacrées aux femmes.

La seconde visite de Wharton commence par le cimetière, lieu de pèlerinage littéraire par excellence ${ }^{46}$. Sand avait souvent manifesté son goût pour « un peu de verdure » (ses derniers mots), ce qui nous ferait imaginer une tombe comme celle de son petit frère Louis racontée dans $H D M V$ (enterré sous un poirier), ou celle de Musset, sous un saule au cimetière du Père Lachaise (figure 2) ${ }^{47}$. Or, elle repose sous une " monstrueuse caisse de pierre » (figure 3$)^{48}$ qui offense la sensibilité de Wharton autant par sa laideur que par son inscription : «la baronne Dudevant ». L’Américaine raille l'incohérence de cette référence au mari répudié qui n'a même pas droit à ce titre et surtout son " inconséquence » pour une "descendante de roi, prêtresse de la démocratie et du fouriérisme », " qui fit porter son pseudonyme illustre par toute la famille »49. Sans savoir que Sand est morte sans laisser aucune instruction et que ses enfants avaient fait ces choix (comme ils avaient célébré des obsèques catholiques que leur mère aurait refusées), la déception entre l'idéal romantique et la réalité bourgeoise marquera le reste de la visite. Elle aurait été encore plus outragée si elle avait su que l'acte de décès de Sand porte la mention « sans profession $» 5^{0}$.

Figure 2. La tombe de Musset. 


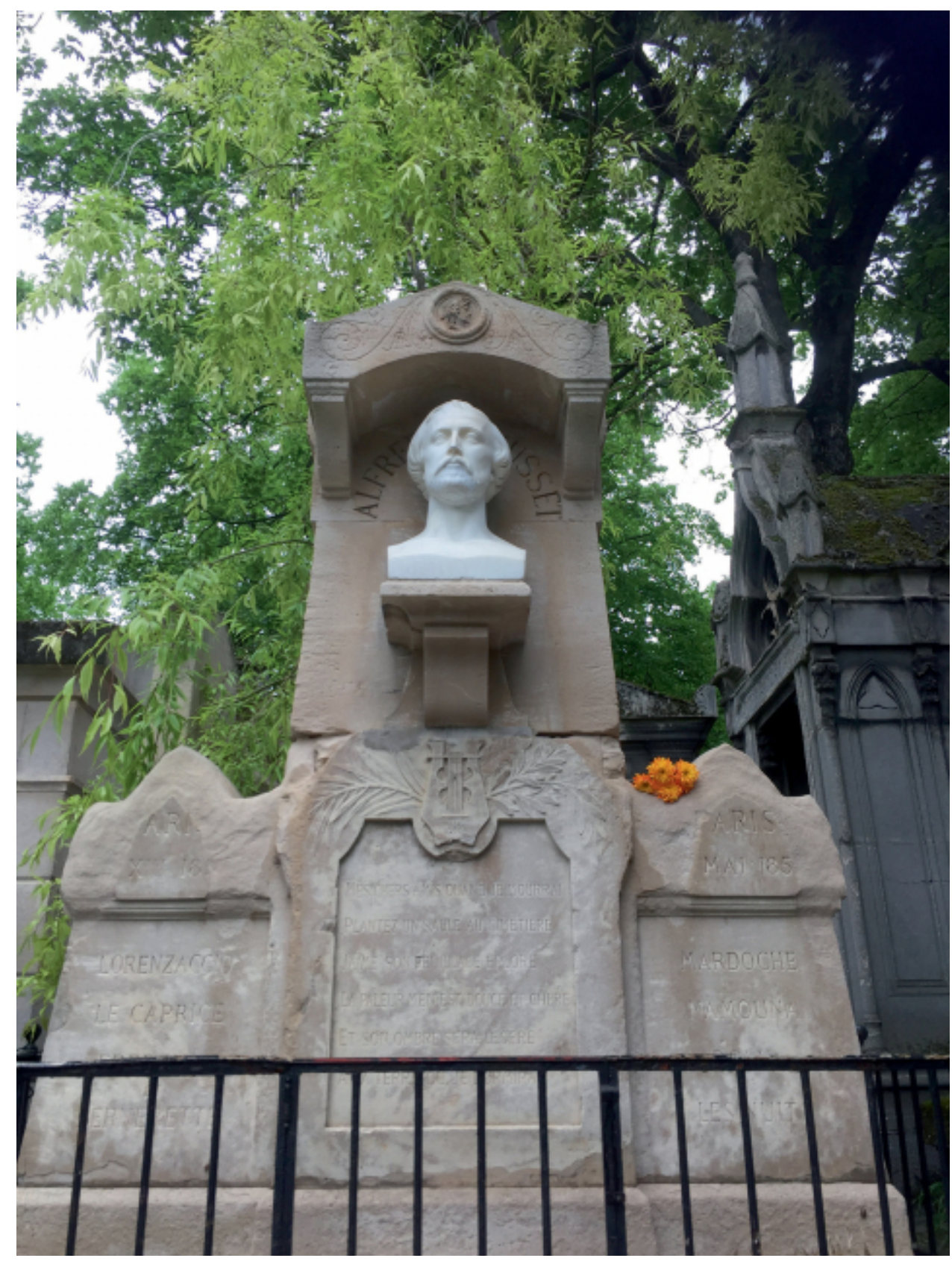

(c) Elizabeth Emery

Figure 3. La tombe de Sand. 


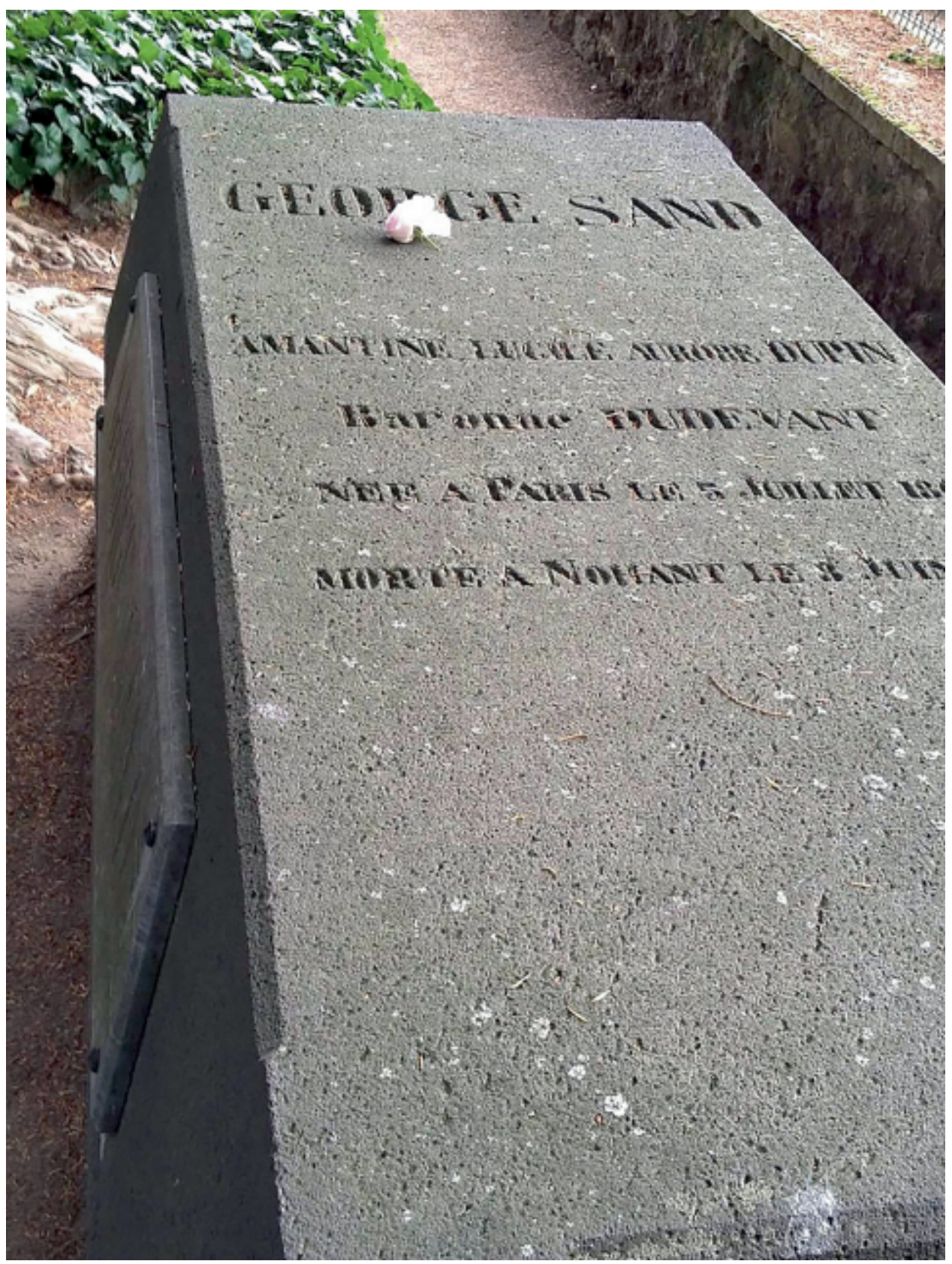

(c) Elizabeth Emery

Wharton a beau écrire que cette prétention bourgeoise n'avait rien à voir avec la vraie vie de Sand, elle trouve peu de traces de la narratrice rebelle de $H D M V$, sans parler de la farouche individualité de ses personnages féminins ${ }^{51}$. Il n'y a que la longue table dans le salon qui lui permet enfin de revivre les scènes de sociabilité à la française qu'elle avait imaginées, celles décrites dans Autour de la table, comme les activités « que l'on peut faire à la campagne, en famille, à travers la causerie, durant les longues veillées de l'automne et de l'hiver $» 5^{2}$. Wharton imagine des visiteurs comme Gautier, Flaubert ou Dumas fils parlant de leurs œuvres pour distraire les autres invités qui peignent, sculptent et cousent (Wharton, 1907 : 81). Ce sont ses souvenirs de lecture qui, projetés sur des pièces vides que fait visiter une bonne, servent d'élément magique qui fait rebattre le cour artistique de la maison.

Cette stratégie consistant à superposer ses souvenirs immatériels de lecture à un espace lors d'une visite de maison-musée est fréquente. Des visiteurs à Lamb House, la résidence d'Henry James à Rye (Sussex, UK), aujourd'hui conservée par le National Trust 53 , remarquent, par exemple, qu'il s'agit d'un musée pour les « fervents admirateurs » (diehard fans), ceux qui connaissent suffisamment bien la vie et l'œuvre de l'écrivain pour projeter leurs souvenirs de lecture sur un lieu autrement peu lisible54. Après tout, la mémoire collective et ses priorités évoluent ; avec le passage du temps, les visiteurs ne reconnaissent pas toujours " l'opérativité symbolique » qui avait initialement inspiré la conservation d'un lieu culturel. 
D'où l'importance de ces récits pour les guides, les audio-guides et la documentation qui expliquent aux visiteurs occasionnels les valeurs patrimoniales qu'on cherche à communiquer. Ce sont des récits qui activent ou réactivent la mémoire collective. La bonne qui sert de guide à Wharton en 1907 révèle, par exemple, qu'elle avait connu Sand et qu'elles ont travaillé ensemble à la fabrication des costumes pour les marionnettes de Nohant. C'est un moment magique où passé et présent se rejoignent par la présence de cette Berrichonne et son amour pour sa maîtresse. C'est l'expérience racontée par la plupart des visiteurs qui résument leur voyage sur TripAdvisor : les guides font surgir le passé dans le présent par leur choix d'anecdotes, donnant au visiteur une impression d'authenticité. Mais la valeur symbolique présentée ne se conforme pas toujours aux attentes du visiteur. Wharton, par exemple, qui cherche les traces de l'écrivain et non de la maîtresse de maison, module le récit de son guide, récupérant la référence à la couture, un métier traditionnel de femme, dans son propre récit pour la relier à l'écriture. Dans A Motor-Flight, ce sont les mêmes « doigts infatigables qui faisaient avancer, en haut, toute la nuit, la plume 55 ».

Les hommes auteurs n'échappent pas eux non plus à la tension entre historicité et littérarité propre à la maison d'écrivain et résumée si bien par une formule sur la page principale de la Maison de Sand : "Une maison en héritage. [...] Une personnalité à redécouvrir ${ }^{6}$. » Comme l'a noté Proust dans « La méthode de Sainte-Beuve », la familiarité engendre le mépris : " un livre est le produit d'un autre moi que celui que nous manifestons dans nos habitudes, dans la société, dans nos vices ». Juger l'écrivain par sa vie ne peut que nuire à ses écrits ${ }^{57}$. En privilégiant la biographie, on mésestime ou ne lit plus - les écrits, substituant à la lecture des œuvres une lecture biographique. Nigel Harkness démontre ce processus pour Henry James. Il admire beaucoup l'œuvre de Sand avant de découvrir ses textes autobiographiques qui le fascinent - d'où le désir de visiter Nohant -, mais il estime en même temps que tant de détails biographiques nuisent à sa réputation littéraire (Harkness, 2016 : 92-93).

28 Cette attention portée à la vie personnelle s'avère particulièrement problématique pour les femmes qui ont déjà du mal à être perçues comme pratiquant un métier qui se fait, souvent de façon imperceptible (« en haut, toute la nuit »). Au contraire de Zola ou d'Hugo, dont l'écriture diurne dictait les activités de la maisonnée (si Hugo demande du papier tout monde court en chercher) ${ }^{58}$, pour Sand les activités domestiques réglaient son écriture, ce qui rendit ce travail largement invisible à ceux qui ont décrit la vie à Nohant 59 . " Écrire à l'ombre des grands hommes », le titre d'un chapitre de La Petite Sœur de Balzac de Christine Planté (2015 : 113-148), résume bien la situation de la plupart des Françaises du XIXe siècle. Même quand elles sont fières de leur travail, elles s'expriment de façon modeste, s'effaçant devant les grands hommes dont dépend leur réussite critique et économique.

Leur position littéraire est semblablement ombrageuse, car seuls les genres mineurs (non littéraires) sont acceptables pour les femmes : romans, lettres, journaux intimes (Planté, 2015 : 199). Puisque ce genre de textes fournit une riche source de détails sur la vie quotidienne ${ }^{60}$, il est facile d'oublier qu'il s'agit de constructions rhétoriques : " Le projet autobiographique se veut entreprise de revendication et de construction identitaire », note Bénédicte Garban, " George Sand se donne forme, se recrée à travers l'écriture » $(2010: 58)^{61}$. Il est donc facile de tomber dans un cercle vicieux qui consiste à renforcer la condition domestique d'une femme à cause du volume de ses écrits traitant de la vie quotidienne, glissant ainsi de George Sand grande écrivaine à George Sand maîtresse de maison, un choix qui renforce, même sans le vouloir, les stéréotypes de genre.

30 Si on prend des textes « bio-fictifs » (Diaz, 2007:42) au pied de la lettre, on risque de minimiser la valeur que leurs auteures leur accordaient, comme le fait Perrot en suggérant que Sand n'écrivait que comme " gagne-pain », qu'elle aurait "préféré un travail plus manuel » et qu'elle n'a jamais, finalement, " fait de la littérature un culte » (2018 : 342). Il est vrai que Sand avait en horreur les institutions littéraires, et surtout leur prétention, mais son rapport au travail domestique et à la production littéraire est beaucoup plus complexe qu'il ne le parait dans ses écrits « bio-fictifs » ${ }^{62}$. Claire White, dans un récent article, analyse la remarquable complexité de la pensée sandienne sur le 
travail littéraire et le travail manuel, tous deux impossibles à distinguer des questions de classe, de prestige, de sexe, d'économie et d'éthique (2018:61-78).

La question de la valeur patrimoniale à communiquer pour la Maison de George Sand est particulièrement importante, car entre la mort de Gabrielle Dudevant-Sand en 1909, le renoncement de l'Académie française au legs (1951), son classement par les Monuments historiques (1952) et l'ouverture de la Maison en 1961 (après la mort d'Aurore Lauth-Sand qui en avait l'usufruit), le monde a bien changé63. Si Wharton et James se préparaient à la visite de la maison de Sand comme à un acte de piété pour rendre hommage à une aïeule littéraire et communier avec son esprit, pour les Parisiens d'après-guerre, la maison de George Sand, de même que ses écrits champêtres comme François le Champi et La Mare au diable, représentaient un mode de vie dévolu : rustique, paysan, berrichon. Déjà en 1912, c'est l'image qui surgit dans un article du Gaulois rapportant une interview avec des domestiques de Sand aux noms bien campagnards («Marie des poules » et « le père Henri ») qui louent Sand comme une « sorte de maman » et « bonne patronne » 64 . Bonniot-Mirloup (2016), dans son étude du livre d'or de la maison, remarque la tendance des visiteurs à chercher un lien affectif avec Sand. Le musée reconnaît ce désir d'intimité dans son slogan : « Au cœur du Berry, entrez dans l'intimité d'un grand écrivain du XIXe siècle : George Sand. »65

Cette évolution de la valeur collective est toute naturelle. Comme le montre Davallon, la « certification », la « déclaration publique » du statut patrimonial d'un objet entraîne la modification de son caractère symbolique (2006 : 96 et 133-135). Lorsqu'une maison privée connue seulement par quelques fervents admirateurs (comme le Nohant-relique - sous " une cloche de verre »- de 1906) rejoint le patrimoine, on cherche à la démocratiser :

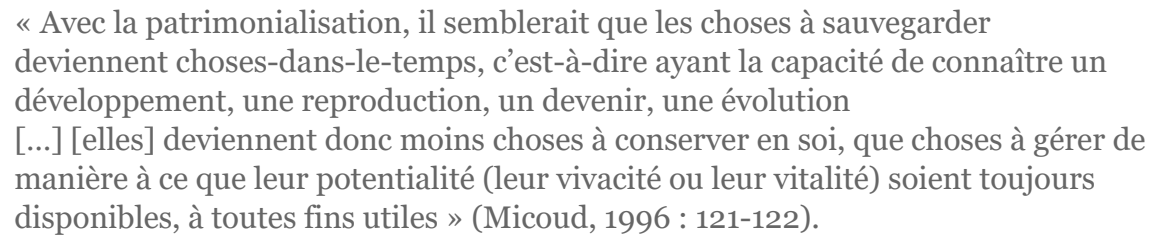

La rénovation de la maison de Zola en « Maison Zola - Musée Dreyfus », prévue pour fin 2019, fournit un bon exemple de ce besoin de gérer cette " potentialité » en proposant une double mission : celle de commémorer à la fois le travail de l'écrivain et celle de son engagement historique dans l'affaire Dreyfus.

Mais cette évolution des « choses à sauvegarder » vers les « choses-dans-le-temps » ne plaît pas toujours aux fervents admirateurs. Henry James remarque déjà la "vulgarisation " de Nohant en 1914, sept ans après la publication de A Motor-Flight. En répondant à une amie anglaise qui souhaite se rendre à Nohant en voiture (il lui recommande d'abord la lecture de $H D M V$ ), il reconnaît que la patrimonialisation transformera un lieu sacré en destination touristique :

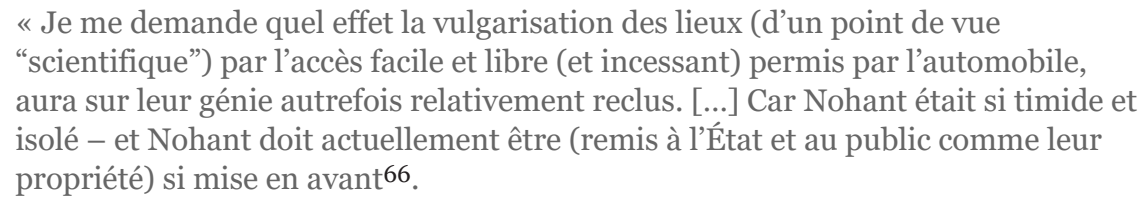

Il recommande l'expérience de façon gardée, rappelant à sa correspondante que la maison est maintenant promise à l'État, ce qui aura sûrement changé sa valeur (son " génie »)67. Il reconnaît que l'exclusivité d'un lieu mémoriel connu seulement de quelques lecteurs de bon goût est incompatible avec la popularisation d'un lieu géré par une institution culturelle ${ }^{68}$. La patrimonialisation nécessite la reconnaissance publique (Davallon, 2016 : 97). D’où la question de conservation soulevée par Saurier (2013) : devrait-on continuer à privilégier la visite intime de quelques visiteurs intéressés par le " lieu de la littérature »? Ou mettre en avant le lieu de vie, accessible au plus grand nombre?

À la Maison de George Sand, on choisit la seconde possibilité : des éléments liés au premier sont présentés à travers le second. Les diverses activités culturelles entreprises 
par la direction visent à informer le public sur les pratiques de la vie quotidienne et la culture matérielle du XIXe siècle - de « Noël en famille chez George Sand » à « La vie quotidienne de George Sand expliquée aux enfants » et à « La collection d'antiquités de Nohant " 99 . L'impressionnante quantité de vestiges authentiques de l'époque de Sand une rareté pour la plupart des maisons d'écrivains - permet des activités qui confèrent à ce musée une vitalité qui répond très bien à la valorisation des " choses-dans-letemps » évoquée par Micoud. À la Maison de George Sand, on fait appel non simplement aux amateurs de la littérature, mais aussi à un public mixte d'enfants, de musiciens, d'écrivains, d'historiens, de collectionneurs et de paysagistes, et bien d'autres encore.

37 Le « lieu de la littérature » n'est pas pour autant négligé. L’usage des réseaux sociaux, pour diffuser les annonces et faire connaître le style de Sand - des citations liées à l'actualité, au temps ou aux activités de la maison -, informe les visiteurs sur la vie de la maison même quand ils habitent loin. C'est une maison d'écrivain qui agit comme un centre culturel ${ }^{70}$. Ces pratiques numériques visant à renforcer les valeurs culturelles du lieu pourraient servir de modèle pour d'autres maisons d'écrivains qui cherchent à être plus visibles et plus vivantes sans encourir de grandes dépenses ${ }^{71}$.

Si la « mémoire de la littérature » passe largement par la mémoire de l'auteur et la mémoire du lecteur (Samoyault, 2004), il faudrait aussi reconnaître que plus l'auteur est éloigné dans le temps, plus le contact entre auteur et lecteur se fait par le biais de textes intermédiaires, que ce soient des anthologies, des éditions abrégées ou des adaptations. A Motor-Flight en est un exemple. Mme Alfred Sutro, la destinataire de la lettre de James ci-dessus, veut visiter la maison de Sand en 1914 après avoir lu $A$ Motor-Flight (James lui envoie une copie de $H D M V$ ). Si la plupart des visiteurs contemporains de Nohant ne préparent plus leur visite par une lecture de Sand, cela ne veut pas dire qu'ils ne se documentent pas autrement. Les commentaires laissés dans TripAdvisor, par exemple, mentionnent d'autres types de connaissance indirecte de son œuvre : des romans, films et émissions télévisées qui mettent en scène le personnage historique, la rebelle dont la vie sociale (et surtout amoureuse) continue, comme au temps de Wharton et James, à fasciner le grand public ${ }^{72}$. On voit un exemple plus récent chez " Thegourmetox », un utilisateur de TripAdvisor qui fait lire Dream Lover d'Elizabeth Berg (2015) à son mari pour préparer leur voyage à Nohant73. Il s'agit d'un roman historique en anglais qui se déroule à Nohant et est narré par Sand elle-même, dont le style est d'ailleurs bien mièvre pour qui sait lire Sand en français. Ce genre de « lecture » intermédiaire a encore plus tendance, aujourd'hui, à se faire par le biais de productions audio-visuelles. On le voit bien avec l'émission "Secrets d'Histoire » (France 2) que Stéphane Bern a consacrée à l'écrivaine en 2016, en partenariat avec la Maison de George Sand. France Bleu constate que cet épisode, le plus regardé de « toute l'histoire de l'émission », " a dopé le tourisme en Berry » en haussant de $38 \%$ le nombre de visiteurs (Bossard, 2016).

Comme A Motor-Flight de Wharton, ce genre de récit ciblant ici le grand public peut servir de moteur à la redécouverte des maisons-musées en attirant l'attention sur leur intérêt culturel pour le plus grand nombre ${ }^{74}$. Mais, comme dans le cas de Sand, où l'emphase est si souvent mise sur sa vie amoureuse (Dream Lover, "Secrets d'Histoire »), il est facile de perdre de vue ses écrits littéraires et politiques. Dans les émissions comme "Une maison, un artiste ", par exemple, il est normal de faire valoir l'aspect patrimonial de la maison. Mais quand on présente Sand comme soumise à l'influence de la maison ( « elle n'aurait pas du tout été l'auteur qu'elle a été [...] s’il n'y avait pas eu cette maison »), on encourt le risque de dévaloriser son génie et surtout son œuvre. "Penser la présentation du patrimoine à partir du public », se demande Davallon, « n'est-ce pas courir le risque de l'instrumentaliser ? » (2006, 33-35). Or, dans la plupart des romans, films et émissions, on privilégie la vision de Sand dans ses rapports affectifs : en amante, hôtesse, mère et maîtresse de maison.

Cette mise en scène de la vie amoureuse et domestique d'une femme soulève de nouveau des questions concernant la politique de la représentation. À quel point tombe-t-on dans des stéréotypes de genre en faisant valoir la vie domestique d'une maison associée à une femme (Sand ou Colette, par exemple) et quelles en sont les conséquences, surtout quand il y a si peu de maisons vouées aux écrivaines 75 ? Pour 
prendre un contre-exemple, Victor Hugo passa quinze années de son exil à Guernesey à décorer et à faire visiter sa maison, Hauteville House, considérée par sa famille comme " un poème domestique ${ }^{76}$. Au lieu de mettre l'emphase sur son jardinage ou ses confitures, comme on le fait chez Sand, on décrit sa direction du chantier 77 . HautevilleHouse est quasiment toujours évoquée dans les termes de la production littéraire hugolienne : « autographe », «poème », « œuvre d'art faite d'œuvres d'art ». La maison est même « lue » comme un livre, notent Chantal Brière et Gérard Audinet ${ }^{78}$. Malgré les incessants chantiers et rénovations dirigés par Sand à Nohant, et récemment mis en valeur par Perrot (2018 : 189-222), on ne parle pas de ces activités comme d'une extension de sa créativité artistique, comme c'est le cas chez Wharton, au « Mount »: "A Great American House Designed by A Great American Writer ». Comme à Hauteville House, les publications de Wharton consacrées au paysagisme et au design sont mises en parallèle avec son œuvre fictive ; elles sont présentées comme des manifestations multidisciplinaires de sa créativité. Le programme « Writers in Residence » - réservé aux femmes - renforce l'aspect littéraire du lieu tout en rehaussant la visibilité des écrivaines contemporaines. C'est le cas de la plupart des maisons d'écrivains consacrés aux hommes, où il s'agit principalement de promouvoir la « littérarité » du grand écrivain par l'exposition ou la conservation des manuscrits et d'autres créations esthétiques79.

\section{Conclusion : maison-musée ou maison d'écrivain?}

41 Représenter l'immatérialité de l'écriture dans une maison d'écrivain constitue un défi majeur, car la matérialité de l'exposition muséale met, par sa nature même, l'emphase sur la vie domestique. Si nous avons tant insisté sur l'importance de rendre plus visible l'écriture des femmes par le biais du cas de Sand, c'est que sa représentation muséale illustre un problème central pour la maison d'écrivain en général : comment réconcilier son double rôle de « lieu de vie » et « lieu de la littérature » (Saurier, 2013:215) ? Si on évacue les aspects proprement littéraires d'une œuvre - la structure et le style - au profit du contenu "bio-fictif ", on encourt le risque d'en renforcer la dimension domestique, ce qui peut faire oublier les productions plus immatérielles de l'écrivain : le contenu même des livres posés sur les étagères. La gestion d'une maison d'écrivain est beaucoup plus compliquée que celle d'une maison-musée en général, car, comme le note Saurier, on doit équilibrer une hétérogénéité de valeurs patrimoniales qui changent d'une maison à une autre et d'une génération à une autre. À la fois " inscription des figures célèbres [...] et espace social de leur construction », elle est un lieu " polysémique », apprécié alternativement par les ouvrages produits dans cette maison (ou consacrés à la maison) et pour sa conservation des vestiges des modes historiques (ibid. : 215-216).

42 Dans cet article, nous avons cherché à mettre en évidence d'une part l'importance de la perspective extérieure (et souvent étrangère) qui justifie la transformation d'une résidence privée en maison-musée, et, d'autre part, le rôle crucial joué par les visiteurs dont les témoignages écrits ou photographiques perpétuent une atmosphère essentielle pour la survie de ces mêmes monuments en tant que lieux de mémoire, qu'ils soient lieux de vie ou lieux de la littérature. Dans une maison d'écrivain, le visiteur cherche à s'inscrire dans une filiation culturelle qui passe le plus souvent par l'imagination, une imagination nourrie par des souvenirs, que ce soit de l'école, de lectures, de films ou d'émissions télévisées, et qui lui permettent, finalement, de former de nouveaux souvenirs (Stewart, 2007 : 133). De La Mare au diable, Dream Lover et "Secrets d'Histoire » aux blogs, selfies et tweets, chaque visiteur, comme Wharton, valide, à sa façon, l'importance de Nohant comme lieu de mémoire et celle de Sand comme figure patrimoniale. 


\section{Bibliographie}

Amirou (Rachid). 1995. Imaginaire touristique et sociabilités du voyage. Paris : PUF.

Audinet (Gérard). 2017. " "Lecture" de Hauteville House ». Communication faite au Groupe Hugo le 21 janvier 2017. En ligne : http://groupugo.div.jussieu.fr/groupugo/17-01-21audinet.htm [consulté le 25 novembre 2019].

Bonniot-Mirloup (Aurore). 2016. «Tourisme et maisons d'écrivain, entre lieux et lettres ». Via, Tourism Review [en ligne], 9 : http://journals.openedition.org/viatourism/795 [consulté le 25 novembre 2019].

DOI : 10.4000/viatourism.795

Bossard (Adèle). 2016. « Stéphane Bern dope le tourisme en Berry ». France Bleu.fr., 13 sept. En ligne : https://www.francebleu.fr/infos/culture-loisirs/l-emission-secrets-d-histoire-booste-lafrequentation-de-la-maison-de-george-sand-1473439722 [consulté le 25 novembre 2019].

Brière (Chantal). 2007. Victor Hugo et le roman architectural. Paris : Champion.

Choay (Françoise). 1992. L’Allégorie du patrimoine. Paris : Seuil.

Davallon (Jean). 2006. Le Don du patrimoine : Une approche communicationnelle de la patrimonialisation. Paris : Hermes Science-Lavoisier.

Delbaere-Garant (Jeanne). 1970. Henry James. The Vision of France. Liège : Presses universitaires de Liège. En ligne : https://books.openedition.org/pulg/895?lang=en [consulté le 25 novembre 2019].

DOI : $10.4000 / 1895.2$

Diaz (Brigitte). 2007. " Poétique de la lettre dans les Lettres d'un voyageur. L'invention d'un style ». Recherches \& Travaux, 70, p. 41-54.

Emery (Elizabeth). 2015. Le Photojournalisme et la naissance des maisons-musées d'écrivains en France (1881-1914). Trad. de l'anglais de Jean Kempf et Christine Kiehl. Chambéry : Université de Savoie Mont-Blanc.

Engelking (Tama). 2018 (à paraître). " Speaking for Colette: literary tourism, storytelling and La Maison de Colette ». Intervention à la conférence internationale "Women in French 2018. Le bruit des femmes », 8-10 février 2018, Florida State University, Tallahassee (FL).

Escholier (Raymond). 1932. Victor Hugo raconté par ceux qui l'ont vu. Paris : Stock.

Garban (Bénédicte). 2010. "Histoire de ma vie de George Sand : création de soi et fabrique du sujet écrivain ", p. 51-61 in Écritures autobiographiques : Entre confession et dissimulation / sous la direction de Jean-Marie Paul \& Anne-Rachel Hermetet. Rennes : Presses universitaires de Rennes.

Garval (Michael). 2004. Dream of Stone: Fame, Vision, and Monumentality in NineteenthCentury French Literary Culture. Newark (DE) : University of Delaware Press.

DOI : 10.1353/lit.2003.0023

Gaviglio-Faivre d'Arcier (Catherine). 2007. Lovenjoul (1836-1907). Une vie, une collection. Paris : Éditions Kiné.

Glaumaud-Carbonnier (Marion). 2018. « La mémoire pérégrine », p. 41-56 in Lire Zola au XXIe siècle / sous la direction de Aurélie Barjonet \& Jean-Sébastien Macke. Paris : Classiques Garnier.

Grandemange (Christophe). 2010. Le Château de Nohant. Maison de George Sand. Saint-Cyrsur-Loire : Éditions Alan Sutton.

Harkness (Nigel). 2016. " "Rather on our shelves than in our hands": remembering George Sand with Henry James ». George Sand Studies, 33-34, p. 77-97.

Hendrix (Harald) (dir.). 2008. Writers' Houses and the Making of Memory. New York : Routledge.

DOI : $10.4324 / 9780203939680$

Hovasse (Jean-Marc). 2008. Victor Hugo. Paris : Fayard, 2 vol.

Hugo (Charles). 1864. Chez Victor Hugo par un passant. Paris : Cadet et Luquet.

James (Henry). 1884. A Little Tour in France. Boston : James Osgood.

James (Henry). 1920. The Letters of Henry James. Édité par Percy Lubbock. New York : Charles Scribner's \& Sons, 2 vol.

Karénine (Wladimir). 1899. George Sand, sa vie et ses œuvres. Paris : Plon, 4 vol.

Lassère (Madeleine). 1997. " Le XIXe siècle et l'invention du tourisme funéraire ». Revue d'histoire moderne et contemporaine, 44(4), p. 601-616.

DOI : 10.3406/rhmc.1997.1887

Lee (Hermione). 2007. Edith Wharton. New York : Knopf.

Levenstein (Harvey). 1998. Seductive Journey: American Tourists in France from Jefferson to the Jazz Age. Chicago : University of Chicago Press. 
Micoud (André). 1996. «Musée et patrimoine : deux types de rapport aux choses et au temps ? ".

Hermès, 20, p. 115-123.

DOI : $10.4267 / 2042 / 14913$

Montandon (Alain) (dir.). 2015. Tissus et vêtements chez les écrivains au XIXe siècle. Sociopoétique du textile. Paris : Champion.

Nora (Pierre) (dir.). 1997. Les Lieux de mémoire. Paris : Gallimard, 3 vol.

Pennell (Joseph). 1925. The Adventures of an Illustrator. Boston : Little, Brown, and Co.

Perrot (Michelle). 2018. George Sand à Nohant. Paris : Seuil (La librairie du XXIe siècle).

Planté (Christine). 2015. La Petite Sœur de Balzac. Lyon : Presses universitaires de Lyon.

DOI : $10.4000 /$ books.pul.22527

Powers (Lyall) (dir.). 1990. Henry James and Edith Wharton. Letters: 1900-1915. New York : Charles Scribner's Sons.

Proust (Marcel). 1971. "La méthode de Sainte-Beuve », p. 219-232 in Contre Sainte-Beuve / sous la direction de Pierre Clarac. Paris : Gallimard (La Pléiade).

Reid (Martine). 2013. George Sand. Paris : Gallimard.

DOI : $10.14375 /$ NP.9782070444014

Ridehalgh (Anna). 1990. "Rousseau as God. The Ermenonville pilgrimages in the Revolution ». Studies on Voltaire and the Eighteenth Century, 278, p. 287-308.

Rocheblave (Samuel). 1905. George Sand et sa fille d'après leur correspondance. Paris : Calmann-Lévy.

Samoyault (Tiphaine). 2004. L'Intertextualité : Mémoire de la littérature. Paris : Nathan.

Sand (George). 1875. Autour de la table. Paris : Michel Lévy frères.

Sand (George). 1964. Correspondance, vol. 14. Édition de Georges Lubin. Paris : Garnier.

Sand (George). 2004. Histoire de ma vie. Édition de Martine Reid. Paris : Gallimard (Quarto).

Saurier (Delphine). 2013. La Fabrique des illustres. Proust, Curie, Joliot et lieux de mémoire. Paris : Éditions Non Standard (SIC : Recherches en sciences de l’information et de la communication).

Schriber (Mary Suzanne). 1991. "Introduction », p. XVII-XLX in Edith Wharton, A Motor-Flight through France. DeKalb : Northern Illinois Press.

DOI : $10.3917 /$ aj.432.0004

Stewart (Susan). 1992. On Longing: Narratives of the Miniature, the Gigantic, the Souvenir, the Collection. Raleigh (NC) : Duke University Press.

Sturgess (Kim). 2004. Shakespeare and the American Nation. Cambridge : Cambridge University Press.

DOI : $10.1017 /$ CBO9781107416321

Trubek (Anne). 2010. A Skeptic's Guide to Writers' Houses. Philadelphie : University of Pennsylvania Press.

DOI : 10.9783/9780812205817

Twain (Mark). 1875. « Proposed Shakespearean Memorial ». New York Times, 29 avril.

Twain (Mark). 1899. "What Barnum did for Shakespeare ", in Following the Equator: A Journey Around the World. New York : Harper Brothers.

Wharton (Edith). 1908. A Motor-Flight Through France. New York : Scribner's Sons.

Wharton (Edith). 1990. A Backward Glance, p. 767-1064 in Edith Wharton. Novellas and Other Writings. Édition de Cynthia Griffin Wolff. New York : Library of America.

Wharton (Edith). 2015. La France en automobile. Trad. de l'anglais par Jean Pavans. Paris : Mercure de France.

White (Claire). 2018. " George Sand, digging », p. 61-78 in The Labour of Literature in Britain and France, 1830-1910: Authorial Work Ethics / sous la direction de Marcus Waithe \& Claire White. Londres : Palgrave.

Withey (Lynne). 1997. Grand Tours and Cooks Tours: A History of Leisure Travel, 1750-1915. New York : William Morrow \& Co.

Wright (Sarah Bird). 1996. Edith Wharton Abroad: Selected Travel Writings, 1888-1920. New York : Palgrave Macmillan.

\section{Notes}

1 Ces vacances en automobile devinrent une tradition de 1904 à 1907. Voir, par exemple, la biographie de Lee (2007 : 273-274). 
2 Ces essais furent publiés une fois par mois de décembre 1906 à février 1907 et de janvier à mars 1908 dans la revue The Atlantic Monthly, et ensuite en volume (Wharton, 1908). La troisième partie du livre publié par Scribner's en 1908 ne fut jamais sérialisée. Toutes les citations en anglais sont traduites par l'auteure.

3 Schriber (1991 : xliv-xlv) et Wright (1996).

4 Les chapitres « La Loire et l'Indre » et « De Paris à Poitiers ».

5 L'autre exception notable vient dans « Des Pyrénées vers la Provence » où elle évoque Mme de Sévigné au château de Grignan.

6 «A pilgrimage to the oracle ». Elle reproduit (en français) la réponse de la patronne de l'Hôtel Sainte-Catherine, à La Châtre, lorsque Wharton lui demande si la maison de Sand se visite : « Le château de George Sand ? (A pause of reflection.) C'est l'écrivain, n'est-ce pas ? (Another pause.) C'est à Nohant le château ? Mais, madame, je ne saurais vous le dire » (Wharton, 1908 : 39). Wharton conclut que la célébrité internationale de Sand ne s'étend pas jusqu'en province : "Fame throws its circles so wide that it makes not a ripple near home » (ibid.).

7 Davallon reconnaît quatre types de « procédures » nécessaires à la patrimonialisation : " l'identification de l'objet » (la découverte de sa valeur) ; "l'authentification du lien avec le passé » (la certification par une institution du statut patrimonial) ; " la déclaration du statut patrimonial de l'objet » (qui modifie son caractère symbolique en indiquant la nécessité de le conserver) ; " l'opérativité symbolique » (la façon de partager avec le public) (2006 : 134-135). « Communiquer le patrimoine » fait partie de la première catégorie (ibid. : 36-38).

8 Choay, 1992 ; Davallon, 2006. Ce n'est pas une simple coïncidence que l'année suivant la seconde visite de Mme Wharton à Nohant (1907), Gabrielle Sand ait légué le château de sa grandmère à l'Académie française afin d'en faire un lieu « d'excursion et de pèlerinage ». La maison fut acceptée provisoirement par l'Académie en 1909 (après la mort de Gabrielle le 27 juin) et officiellement en septembre 1912. Sa sœur, Aurore Lauth-Sand, en aura l'usufruit jusqu'à sa mort en 1961.

9 Ridehalgh (1990), Withey (1997) et Hendrix (2008 : 15-29) évoquent le culte des pèlerinages littéraires de la fin du XVIIe siècle au XIXe siècle.

10 Voir, par exemple, Garval (2004) et Perrot (2018). Wladimir Karénine est le pseudonyme de Varvara Dmietrievna Stasova Komarova (1862-1942). Aujourd'hui, la popularité de romans et films ciblant un public anglophone maintient cette renommée internationale : Dream Lover d'Elizabeth Berg (2015), Impromptu de Julian Sands (1991), parmi d'autres.

11 Nigel Harkness (2016) et Jeanne Delbaere-Garant (1970) résument l'évolution de l'attitude de James envers Sand.

12 Wharton et James lisent des romans comme La Mare au diable et parlent dans leurs lettres de différents ouvrages consacrés à Sand (comme ceux de Karénine [1899] et Rocheblave [1905]). Ils se les envoient, avec des passages qui les intéressent indiqués au crayon (voir Powers, 1990:53 et 215, par exemple).

13 https://www.edithwharton.org/ (consulté le 25 novembre 2019).

14 Powers évoque ces surnoms en termes d'érotisme (1990:13-16) lorsque Wharton elle-même explique dans ses mémoires leur comédie (Wharton, 1990 : 897-898 et 916).

15 Sand, $1964: 405$.

16 Wharton note que James avait partagé avec elle des réminiscences de Nohant racontées par Flaubert et Maupassant lors de ses tous premiers séjours parisiens dans les années 1870 (Wharton, 1990).

17 "I've really been for weeks in the disabled state, with the bleeding wound in my side, produced by the Parthian shot of your own last - your fling back at me, over your departing shoulder, of your unutterable vision of the Nohant that I have all these (motorless) years so abjectly failed to enlighten my eyes withal [...] the golden mist of your Nohant [...] the very first question on which I shall beset you will be your adventure and impression of Nohant - as to which I burn and yearn for fond particulars. [...] To think that you have seen La Châtre! [...] But it [the javelin from my side] will really stick there, poisoning my blood, till you write - I mean till you print, till you "do" the place, the whole impression for me under stress of imminent publication. For of course you are doing, you have done that. You can't not. I yearn \& languish. Write to me that this act of piety is even already performed. [...] There has been, you know, no récit (of the impression of the place) of any sort of authority or value but George's own. How you must have smelt them all! » (Powers, 1990 : 65-66). L'emphase est de James.

18 Lettre à Mme Alfred Sutro du 28 juillet 1914 (James, 1920 : II, 375).

19 Sand (2004 : 667 sqq.), Perrot (2018 : 101). Le chemin de fer n'arrive à Nohant-Vicq qu'à la fin du XIXe siècle, à temps pour accueillir des visiteurs venus pour le centenaire de Sand en 1904.

20 Deux ans plus tard, il parlera des maisons d'écrivains en tant que «lieux saints »: "What shrines we do arrange for ourselves! » (lettre à Wharton du 3 avril 1908, Powers, 1990 : 98).

21 Le texte fut d'abord intitulé En Province et sérialisé dans The Atlantic Monthly (1883-1884) avant d'être repris en volume en 1884 (James, 1884), puis réédité avec de nouveaux passages en 
1901. Schriber a compté les 600 guides touristiques consacrés à la France pour un lectorat angloaméricain au XIXe siècle (1991: XXXIX).

22 « The best guidebook to that country I know » (Pennell, $1925: 265)$.

23 « Historique » de la Fédération : http://www.litterature-lieux.com/page-historique.htm (consulté le 25 novembre 2019).

24 Lettre du 17 novembre 1906 (Powers, 1990 : 67).

25 Voir Lee (2007 : 228-229) pour les étapes de ce voyage.

26 C'est un semblable acte de pèlerinage - suivre le chemin de Wharton - qui a inspiré l'édition critique de A Motor-Flight (Schriber, 1991 : XI).

27 Rachid Amirou évoque l'aspect « spirituel » de la maison d'écrivain : « un sentiment diffus d'imprégnation par les lieux [...] une sorte de croyance en une possible rencontre intime avec l'“esprit” du mort qui hanterait l'endroit [...] qui ferait du visiteur le dépositaire imaginaire d'un génie littéraire » (1995: 70).

28 Il s'agit d'un passage des Lettres d'un voyageur cité par Perrot (2018 : 98). Bien qu'il s'agisse d'un narrateur « bio-fictif » (Diaz, 2007 : 42), Perrot note que c'est Sand elle-même qui cherche à renforcer cette idée de Nohant comme centre culturel, invitant des amis et essayant d'établir une communauté d'artistes (2018: 97-134).

29 «When one considers the fatigue of the long day's railway journey, and the French dread of déplacements, the continual stream of greatness that Paris poured out upon Nohant gives the measure of what Nohant had to offer in return » (Wharton, 1908: 39).

30 Sur l'emplacement de l'actuel hôtel de ville.

31 Voir, par exemple, le site officiel du tourisme au pays de George Sand, à La Châtre : http://www.pays-george-sand.fr/fr/ (consulté le 25 novembre 2019). L’Association des amis de la route historique George Sand fut établie en 1999.

32 "She formed, at any rate, a charming link between our imagination and the famous house; and we presently found that the miracle which had preserved her in all her 1830 grace had been extended to the whole privileged spot, which seemed, under a clear glass bell of oblivion, to have been kept intact, unchanged, like some wonderful "exhibit" illustrative of the extraordinary history lived within it » (Wharton, $1908: 42$ ).

33 "The delicate and hermetic world of the souvenir is a world of nature idealized; nature is removed from the domain of struggle into the domestic sphere of the individual and the interior » (Stewart, $1992: 145)$.

34 Sa représentation de Nohant fait penser aux écrits de son contemporain Aloïs Riegl, discutés par Davallon, et surtout le monument historique comme « témoin du passé » : «L'édifice par sa présence est le représentant du contexte historique, social, matériel, humain auquel il a appartenu et auquel nous n'avons accès que par lui » (Davallon, 2006 : 88).

35 Elle n'est pas seule. Ce sont les conclusions de quasiment tous ceux qui discutent le fonctionnement affectif de la maison d'écrivain (par ex. Saurier, $2013: 215-216$ ).

36 « Does a sight of Nohant deepen the mystery, or elucidate it? [...] Both. For if it helps one to understand one side of George Sand's life, it seems actually to cast a thicker obscurity over others » (1908: 44).

37 Voir une lettre de James du 28 juillet 1914 qui donne des conseils à une amie qui entreprend le voyage.

38 Il avait formalisé son vœu dans un testament de 1905 (Gaviglio-Faivre d'Arcier, 2007 : 185196).

39 Gaviglio-Faivre d'Arcier (2007 : 183-186). D'autres dons à l'Institut ont suivi, notamment la Fondation Thiers (1905) et l'actuel Musée Jacquemart-André, annoncé dans la presse à partir de la fin des années 1890 et accepté en 1912.

40 Pour les rapports entre Gabrielle et Aurore Sand et Lovenjoul, voir Gaviglio-Faivre d'Arcier (2007). Un article dans le Gil Blas du 4 juillet 1909 rapporte les paroles d'Aurore qui explique que sa sœur avait souhaité qu'on en fasse « un musée où le visiteur retrouverait tous les souvenirs de jeunesse de l'écrivain et ses portraits de famille ».

41 Voir le compte rendu du procès suivant le testament, publié dans le Recueil de la Gazette des tribunaux le 16 octobre 1910, p. 443-446. Je n'ai pas encore trouvé de correspondance prouvant ce lien entre Bourget et les Sand, mais le calendrier serait logique vu l'amitié entre Wharton et Bourget, sa visite à l'Académie, et la correspondance entre Lovenjoul et plusieurs académiciens.

42 La longue histoire de la maison de Zola et du financement des rénovations montre la précarité économique des lieux littéraires même les plus reconnus. Pour l'importance des discours, voir Glamaud-Carbonnier (2018).

43 On parle du « cœur » de ses activités dans des entretiens réalisés pour l'émission « Une maison, un artiste » conçue et diffusée par France 5 en 2016.

44 Wharton se plaint dans ses lettres du manque de temps pour écrire lorsqu'elle est en déplacement. La vente de sa maison chérie («The Mount ») en 1911 se fait avec grand regret (Lee, 
2007 : 387-392). Perrot explique les maintes responsabilités de Sand à Nohant, des domestiques au fonctionnement de l'équipement de la maison et des terres (2018 : 151-175, 191-220 et 259283).

45 " The shrine of those household pieties to which [...] the devotion of her last years was paid » (Wharton, $1908: 47$ ).

46 Voir, par exemple, Lassère (1997).

47 Sand, comme Musset, préfère la verdure : « Je déteste les monuments et les inscriptions [...] les arbres et les fleurs sont les seuls ornements qui n'irritent point la pensée » (HDMV:621).

48 «Monstrous stone chest» (Wharton, 1908 : 79).

49 "She who had made her pseudonym illustrious enough to have it assumed by her whole family should cling in death to the obscure name of a repudiated husband [...] more inconsequent still that the descendant of kings, and the priestess of democracy and Fourierism, should insist on a right to the petty title [barony] which was never hers, since it was never Dudevant's to give! » (Wharton, $1907: 8$ ).

50 Voir Perrot (2018 : 393 et 397-398) et Reid (2013: 206-209) pour les débats familiaux concernant cette tombe et les noms et éléments symboliques choisis.

51 Il est difficile de savoir ce qu'elle aurait vu exactement. Après la mort de Maurice Sand en 1889, la famille a mis en vente la maison (ils n’ont pas trouvé d'acheteur), dispersé le mobilier dans des ventes qui ont duré treize jours, et dispersé les 8000 volumes de la bibliothèque à Paris en 1890 (Perrot, 2018 : 401-402).

52 « French sociability », « a mental table d'hôte » (Wharton, $1908: 81$ ). « Elle a prêté son dos patient à tant de choses ! Écritures folles ou ingénieuses, dessins charmants ou caricatures échevelées, peinture à l'aquarelle ou à la colle, maquettes de tout genre, études de fleurs d'après nature [...] préparations entomologiques, cartonnage, copie de musique, prose épistolaire de l'un, vers burlesques de l'autre, amas de laines et de soies de toutes couleurs pour la broderie, [...] que sais-je ? Tout ce que l'on peut faire à la campagne, en famille, à travers la causerie, durant les longues veillées de l'automne et de l'hiver »(Sand, $1875: 2$ ). Ce passage est toujours cité dans la fiche de visite du Domaine de George Sand à Nohant (http://www.maison-georgesand.fr/view/pdf/648270 [consulté le 25 novembre 2019]).

53 https://www.nationaltrust.org.uk/lamb-house (consulté le 25 novembre 2019).

54 " [...] if you are a diehard Henry James fan, you can always imagine your way through those three rooms. Otherwise, the exhibit may be something of a disappointment for the casual visitor » (commentaire du 19 mai 2003 : https://www.tripadvisor.com/ShowUserReviewsg186276-d218380-r1023714-Lamb_House-Rye_East_Sussex_England.html [consulté le 25 novembre 2019]). Le site Web du musée annonce pour l'été 2019 une " réécriture » ( Rewriting Lamb House ») qui rendra son histoire plus visible.

55 « The indefatigable fingers which drove the quill all night upstairs » (1907 : 83). Elle lie aussi la couture au contexte des activités artistiques du projet collectif du théâtre de Nohant qu'elle décrit avec enthousiasme.

56 http://www.maison-george-sand.fr/ (consulté le 25 novembre 2019).

57 Proust, 1971 : 221-222.

58 Dans Chez Victor Hugo, on décrit la performativité sociale d'une scène où Hugo veut dessiner : " il envoie chercher dans la maison du papier, une plume, de l'encre ; on court partout, on monte dans les chambres, on furette, on fouille, et enfin au bout d'un quart d'heure on rapporte un encrier desséché, une plume qui ouvre le bec et un morceau de papier quelconque » (1864 : 32). Sur le thème de la couture chez Sand, voir Simone Bernard-Griffiths, "Sociopoétique du vêtement dans l'imaginaire sandien » (dans Montandon, 2015 : 49-65).

$59 \mathrm{Si}$ cette tradition d'écriture nocturne avait comme instigateur les circonstances familiales, Sand elle-même a choisi de la continuer une fois les enfants grandis (Perrot, 2018 : 341-349).

6o Perrot raconte, par exemple, l'utilité des écrits de Sand pour la composition du volume IV de L'Histoire de la vie privée, qu'elle a dirigé en 1987 (2018 : 10-11).

61 Pour un exemple au masculin, pensons à la lettre de James à Wharton concernant son premier voyage à Nohant. Il l'avait composé de façon très littéraire (références mythologiques, beau style) sachant qu'elle la publierait.

62 Voir Planté, 2015 : 149-180.

63 Grandemange évoque les nombreuses péripéties concernant le statut patrimonial de la maison (2010 : 118-144).

64 Voir un article dans Le Gaulois du 21 septembre 1912, sous la rubrique « Échos de partout ».

65 Sous-titre affiché actuellement sur le site Web de la maison-musée : http://www.maisongeorge-sand.fr/ (consulté le 25 novembre 2019).

66 "I ask myself what effect the vulgarization of places, "scientifically" speaking, by free and easy (and incessant) motor approach may be having on their once comparatively sequestered genius. [...] For Nohant was so shy and remote - and Nohant must be now (handed over to the 
State and the Public as their property) so very much to the fore » (lettre du 28 juillet 1914 à Mme Sutro).

67 Cette ambivalence est tout de même ironique quand nous savons que ce fut James lui-même qui encouragea Wharton à « faire le lieu », à communiquer son importance culturelle.

68 Saurier évoque les difficultés de plaire à ces deux publics si différents (2013 : 43-44 et 215).

69 https://www.my-loire-valley.com/2017/12/fetez-noel-en-famille-poussez-la-porte-de-lamaison-de-george-sand/ (consulté le 25 novembre 2019).

70 La variété d’activités affichée sur la page Facebook de la maison témoigne de cette remarquable vivacité multidisciplinaire

https://www.facebook.com/pg/MaisonDeGeorgeSandANohant/events.

71 Leur compte Facebook, par exemple, rend Sand actuelle en choisissant d'afficher des citations liées à l'actualité, au temps ou aux activités de la maison. Leurs traductions vers l'anglais sont malheureusement très approximatives (traductions de machine ?), et l'on souhaiterait qu'ils rendent mieux hommage au style de Sand en recrutant des traducteurs anglophones, ne serait-ce que des bénévoles.

72 Voir le sous-titre de l'épisode d' « Une maison, un artiste » (France 5) dédié à « la rebelle de Nohant » en 2016.

73 TripAdvisor le 9 mai 2018 : https://www.tripadvisor.com/Attraction_Review-g1919397d230771-Reviews-George_Sand_s_House-

Nohant_Vic_Indre_Berry_Centre_Val_de_Loire.html (consulté le 25 novembre 2019).

74 Levenstein (1988) décrit bien l'explosion du tourisme automobile des premières années du XXe siècle.

75 Voir, par exemple, Engelking (2018) qui montre l'importance accordée à la voix de Colette et ses expériences domestiques dans la Maison de Colette à La Puisaye, ou la maison de Marguerite Yourcenar (Petite Plaisance) à Northeast Harbor, Maine (États-Unis), vouée à conserver en relique le cadre domestique à l'intérieur duquel l'écrivain a produit ses ouvrages les plus connus.

76 Hugo décrit ses travaux domestiques dans les lettres à Sand et l'encourage à venir lui rendre visite. Elle n'arrive pas à s'y déplacer, Guernesey étant encore plus excentré que Nohant. Voir Hovasse (2008 : 494).

77 Ce sont les détails qu'il avait tendance à enregistrer dans ses cahiers (son épouse et sa fille se sont occupées des détails domestiques).

78 Ces associations sont surtout promues dans Chez Victor Hugo par un passant, un livre diffusé de façon anonyme mais en réalité écrit par Charles Hugo. Voir Audinet (2017) et Brière (2007 : 491-521).

79 C'est aussi un choix voulu par le fonds de ces musées : la plupart des manuscrits sandiens se trouvent dans des bibliothèques et archives parisiens, lorsque la maison de Balzac possède peu d'objets matériels ayant appartenus à Balzac. La villa Marguerite Yourcenar, à Saint-Jans-Cappel, dans le Nord, suit ce modèle : à la fois résidence d'écrivains et un centre culturel, on y met l'accent sur la littérature.

\section{Table des illustrations}

\begin{tabular}{|c|c|c|}
\hline & Titre & Figure 1. Le pays de George Sand. \\
\hline & its & (c) Domaine public. \\
\hline 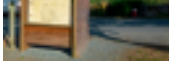 & Uي & $\begin{array}{l}\text { http://journals.openedition.org/culturemusees/docannexe/image/3819/img- } \\
\text { 1.jpg }\end{array}$ \\
\hline & Fichier & image/jpeg, 628k \\
\hline & Titre & Figure 2. La tombe de Musset. \\
\hline & Crédits & (c) Elizabeth Emery \\
\hline & URL & $\begin{array}{l}\text { http://journals.openedition.org/culturemusees/docannexe/image/3819/img- } \\
\text { 2.jpg }\end{array}$ \\
\hline & Fichier & image/jpeg, 1,2M \\
\hline & Titre & Figure 3. La tombe de Sand. \\
\hline & Crédits & (c) Elizabeth Emery \\
\hline & Uي: & $\begin{array}{l}\text { http://journals.openedition.org/culturemusees/docannexe/image/3819/img- } \\
\text { 3.jpg }\end{array}$ \\
\hline & Fic & image/jpeg, 1,2M \\
\hline
\end{tabular}

\section{Pour citer cet article}


Référence papier

Elizabeth Emery, « Un « pèlerinage à l'oracle » : Edith Wharton, Henry James et la

patrimonialisation de la maison de George Sand à Nohant », Culture \& Musées, 34 | 2019, 107 138.

Référence électronique

Elizabeth Emery, «Un « pèlerinage à l'oracle » : Edith Wharton, Henry James et la patrimonialisation de la maison de George Sand à Nohant », Culture \& Musées [En ligne],

34 | 2019, mis en ligne le 16 décembre 2019, consulté le 08 juin 2021. URL :

http://journals.openedition.org/culturemusees/3819; DOI :

https://doi.org/10.4000/culturemusees.3819

\section{Auteur}

\section{Elizabeth Emery}

Université d'État de Montclair

Elizabeth Emery est professeure à l'Université d'État de Montclair (New Jersey, États-Unis). Elle a publié plusieurs livres portant sur la culture française du XIXe siècle, parmi lesquels :

Romancing the Cathedral: Gothic Architecture in Fin-de-siècle French Culture, 2001 ; Consuming the Past: The Medieval Revival in Fin-de-siècle France (dir., avec Laura Morowitz), 2003 ; Le Photojournalisme et la naissance des maisons-musées d'écrivains en France (1881-1914), 2016 ; En toute intimité... Quand la presse people de la Belle Époque s'invitait chez les célébrités, 2015 ; Reframing Japonisme: Women and the Art Market in Nineteenth-Century France, 2019.

Courriel : emerye[at]mail.montclair.edu

\section{Droits d'auteur}

Culture \& Musées 